\title{
A FAST SIGNAL-DEPENDENT TIME-FREQUENCY REPRESENTATION
}

\author{
Yimin Lin \\ Department of Physics, Shantou University \\ Shantou, Guangdong, P.R. China \\ F. H. Y. Chan, F. K. Lam \\ Department of Electrical and Electronic Engineering \\ The University of Hong Kong, Hong Kong
}

\begin{abstract}
In last few years, in order to overcome some limitations of the short time Fourier transform (STFT), while avoiding the cross-terms that make the Wigner distribution difficult to interpret, some signal-dependent time-frequency representations(SDTFR) have been proposed. In this paper, we introduce a computationally efficient signal-dependent time-frequency method which is suitable for on-line analysis. This SDTFR uses a Gaussian window (GW) similar to STFT, but varies the parameter $\sigma$ of the GW with time to achieve high signal concentration and high resolution in time. The parameter $\sigma$ can be automatically calculated by the slope of instantaneous frequency (IF) and instantaneous bandwidth (IB) at that time.
\end{abstract}

\section{INTRODUCTION}

In signal theory, either time or frequency analysis alone is not good enough for nonstationary signal, because each one does not fully describe what is happening. To obtain timevarying information, it is necessary to study the signal density simultaneously both in time and frequency. The most familiar TFR method is the STFT [1]. There is an inherent trade off between time and frequency resolution in STFT. Other time-frequency representations (TFR) that overcome the limitation of the spectrogram have been developed. The first of these was the distribution proposed by Wigner [2]. The inherent bilinear structure of Wigner distribution causes undesirable interfering cross terms.

In 1993, Baranink [3] proposed an optimal-kernel SDTFR. In his methods, in order to obtain a good performance, a weighting function must be designed such that it can pass the auto-components and suppress the crosscomponents in the ambiguity plane. Since different signal gives different ambiguity function, and for each signal, a weighting function must be designed to filter the crosscomponents. In his method, he proposed a new procedure for selecting a signal-dependent weighting function.

In this paper, we present a simple computationally efficient scheme for computing a single parameter for a TFR. This TFR is called fast signal-dependent timefrequency representation (FSDTFR). This method is very suitable for band-limited signal and can be used for real-time analysis. The basic idea of this representation is very similar to the STFT, but the window is changed with the change of signal. If the signal is very stable, then we can use a long window. If the signal is fast changing, then we must use a short window. In other words, the window must be changed with the change of the signal. IF and IB can well be used to describe the change of the signal. A new FSDTFR which uses a Gaussain window similar to STFT, but varies a single parameter $\sigma$ of Gaussian window can be automatically calculated by the slope of the IF and IB at that time.

\section{METHODS}

The short-time Fourier transform, defined as

$$
\operatorname{STFT}(t, w)=\int_{-\infty}^{+\infty} s(\tau) h(\tau-t) e^{-j 2 \pi f \tau} d \tau
$$

in which $s(t)$ is the signal and $h(t)$ is the window function Window function can be considered as a window that selects a particular portion of the signal centered around the given time location, and the Fourier transform of the windowed signal yields the frequency content of the signal at the given time. Gabor and Janssen [4] found that Gaussian window achieves minimum time-frequency uncertainty.

Since the Gaussian window achieves minimum timefrequency uncertainty, in my time-frequency method, GW which is the normalized Gaussian time window

$$
\frac{1}{\sqrt{\pi} \sigma(t)} e^{-t^{2} / \sigma^{2}(t)}
$$

will be used. The basic idea of my FSDTFR is similar to the STFT, but the shape of the GW is change with the change of signal. The change of the signal can be described by IF and IB. In other words, the parameter $\sigma$ of the GW is reiated to the IF and IB. Barber and Ursell [5] have determined the optimal bandwidth of the window for signal with timevarying frequency to be roughly equal to the square root of the time derivative of the IF of the signal.

In order to maintain the basic frequency resolution, the parameter $\sigma$ can only change between the largest value and the smallest value which depend on the basic frequency resolution. When the time derivative of the IF and IB are 
large, corresponding to the fast changing of the signal, then small parameter $\sigma$ corresponding to the sharpening of the GW must be used. But how does the parameter $\sigma$ change with the derivative of IF and IB between the largest value and smallest value? We choose that parameter $\sigma$ changing with the derivative of IF and IB by exponential function which is simple and easily understood between those two value. We define the parameter $\sigma(t)$ of $\mathrm{GW}$ in terms of

$$
\sigma(t)=A e^{-B\left(\frac{d}{d} f_{i}+\frac{d}{d} f_{b w}\right)^{1 / 2}}+C
$$

where $f_{i}$ is the IF. It was yielded by calculation of the first moment with respect to frequency

$$
f_{i}=\frac{\int f \rho(t, f) d f}{\int \rho(t, f) d f}
$$

Since the IF is an averaged quantity, IB seems reasonable to inquire about its standard deviation, or spread. The classical bandwidth definition is the spread of frequencies about the average given by

$$
f_{b w}^{2}=\frac{\int\left(f-f_{i}(t)\right)^{2} \rho(t, f) d f}{\int \rho(t, f) d f}
$$

in equation (3), $A, B$ and $C$ are constants. $C$ is the smallest value of parameter $\sigma$ of the $\mathrm{GW}$. Constant $\mathrm{A}+\mathrm{C}$ is the largest value of the parameter $\sigma$. A fast signal-dependent time-frequency representation is defined as

$$
\text { FSDTFR }=\int_{-\infty}^{+\infty} s(\tau) h(t-\tau) \frac{1}{\sqrt{\pi} \sigma(t)} e^{-t^{2} / \sigma^{2}(t)} e^{-j 2 \pi t} d \tau
$$

where $s(\tau)$ is the signal, $h(t-\tau)$ is the rectangular window.

In equation (3), $\mathrm{C}$ is chosen depending on the basic frequency resolution which depend on the width of the rectangular window and the sampling frequency of the signal. When $C$ is established, the biggest $\sigma$ is depending on the constant A. The highest sidelobe level of the $G W$ is depending on the Gaussian parameter $\sigma$. In general, $\sigma$ must be smaller than 0.3 . In our experiment, we set the largest parameter $\sigma$ as 0.14 which means the constant $A$ is equal to 0.10 . If a large constant $B$ is chosen in equation (3), then parameter $\sigma$ changes quicker to the smallest value with the derivative of IF and IB. If constant $B$ is small, then, parameter $\sigma$ changes slowly. In our study, $B=0.4$ has been chosen for our testing signal and biomedical signal.

\section{RESULT}

Different testing signal was used to compare the performance of different TFR, including STFT, WignerVille, Baranink's TFR and our FSDTFR. The testing results [6-7] show that STFT is incapable of providing high resolution simultaneously in time and frequency. The analysis results using Wigner-Ville method are highly concentrated, but the cross-terms between the various components make this plot difficult to interpret. Although there are some cross-components in testing result using Baranink's method, but the cross-components energy is very small and the auto-components are highly concentrated.

Our FSDTFR is superior to the fixed window (with fixed parameter $\sigma$ ) STFT. Although the auto-components are less concentrated than in the Wigner-Ville method, the FSDTFR lacks the cross-components. This FSDTFR has been successfully used to analyze the Precordial Doppler Ultrasound signal for detecting venous air embolism [7]

\section{CONCLUSIONS}

We presented a simple, computationally efficient TFR. A single parameter depends on the time derivative of IF and IB as in equation (3). In this equation, Constant $\mathrm{C}$ is selected depending on the basic frequency resolution. Constant A relates to the minimizing sidelobe level, and constant $B$ is the sensitivity of parameter $\sigma$ changing with the time derivative of IF and IB. The results showed that FSDTFR gave better performance than others. Since the FSDTFR requires a simple procedure which requires only two or three times the computational cost of a fixed-window STFT to automatically calculate the parameter of the window, it is suitable for use in real-time application. In this study, we have successfully used this method to analyze Doppler heart sound signal and surface EMG signal [6-7].

\section{REFERENCES}

[1] Michael 'R.P., "Time-frequency Representation of Digital Signals and System Based on Short-time Analysis," IEEE Trans. on ASSP, vol.ASSP-28, No.1, pp.55-69, Feb. 1980.

[2] Wigner E., "On the Quantum Correction for Thermodynamic Equilibrium," Phys: Rew., 40, pp.749759, 1932.

[3] Baranink R.G., and Jones D.L., "A Signal-dependent Time-frequency Representation: Optimal Kernel Design," IEEE Trans. on Signal Processing, vol.41, No.4, pp1589-1602, April 1993.

[4] Janssen A.J.E.M., "Optimality Property of the Gaussian Window Spectrogram," IEEE Trans on Signal Processing, vol.39, No.1, pp.202-204, Jan. 1.991.

[5] Barber N.F., Ursell F., "The Response of a Resonant System to a Gliding Tone," Phil. Mag., Vol.39, pp.345361, 1984.

[6] Y.M.Lin, "Application of Time-frequency Method in Venous Air Embolism Detection and in the Study of EMG Fasciculation," Ph.D Thesis, Dept. of Electrical and Electronic Engr. The Univ. of Hong Kong., pp.234-2-53, 1994.

[7] P.W. Lui, Y.M.Lin et.al, "Spectral Characteristics of Embolic Heart sounds Detected by Precordial Doppler Ultrasound During Venous Air Embolism in Dogs," British Joumal Anaesthesia. 71:pp.689-695, 1993. 